\title{
DETECTION OF VERY HIGH ENERGY $\gamma$-RAY EMISSION FROM THE PERSEUS CLUSTER HEAD-TAIL GALAXY IC 310 BY THE MAGIC TELESCOPES
}

J. Aleksić1 ${ }^{1}$, L. A. Antonelli ${ }^{2}$, P. Antoranz ${ }^{3}$, M. Backes ${ }^{4}$, J. A. Barrio ${ }^{5}$, D. Bastieri ${ }^{6}$, J. Becerra González ${ }^{7,8}$, W. BednareK ${ }^{9}$, A. Berdyugin ${ }^{10}$, K. Berger ${ }^{7}$, E. Bernardini ${ }^{11}$, A. Biland ${ }^{12}$, O. Blanch ${ }^{1}$, R. K. Bock ${ }^{13}$, A. Boller ${ }^{12}$, G. BonNOLI ${ }^{2}$, P. Bordas ${ }^{14}$, D. Borla Tridon ${ }^{13}$, V. Bosch-Ramon ${ }^{14}$, D. Bose ${ }^{5}$, I. Braun ${ }^{12}$, T. Bretz ${ }^{15}$, M. Camara ${ }^{5}$, A. Cañellas ${ }^{14}$, E. Carmona ${ }^{13}$, A. Carosi $^{2}$, P. Colin $^{13}$, E. Colombo ${ }^{7}$, J. L. Contreras ${ }^{5}$, J. Cortina ${ }^{1}$, L. Cossio ${ }^{16}$, S. Covino ${ }^{2}$, F. Dazzi ${ }^{16,30}$, A. De Angelis ${ }^{16}$, E. De Cea del Pozo ${ }^{17}$, B. De Lotto ${ }^{16}$, M. De Maria ${ }^{16}$, F. De Sabata ${ }^{16}$, C. Delgado Mendez ${ }^{7,31}$, A. Diago Ortega ${ }^{7,8}$, M. Doert ${ }^{4}$, A. Domínguez ${ }^{18}$, D. Dominis Prester ${ }^{19}$, D. Dorner ${ }^{12}$, M. Doro $^{6}$, D. Elsaesser ${ }^{15}$, M. Errando ${ }^{1}$, D. Ferenc ${ }^{19}$, M. V. FonseCA ${ }^{5}$, L. FonT ${ }^{20}$, R. J. García LóPez $Z^{7,8}$, M. GarczarczyK ${ }^{7}$,

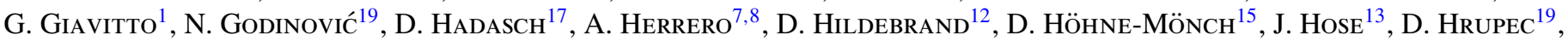

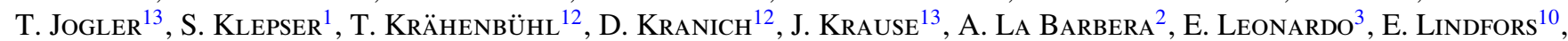

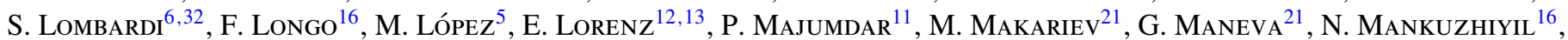
K. Mannheim ${ }^{15}$, L. Maraschi ${ }^{2}$, M. Mariotti ${ }^{6}$, M. Martínez ${ }^{1}$, D. Mazin ${ }^{1}$, M. Meucci ${ }^{3}$, J. M. Miranda ${ }^{3}$, R. Mirzoyan ${ }^{13}$, H. Miyamoto ${ }^{13}$, J. Moldón ${ }^{14}$, A. Moralejo ${ }^{1}$, D. Nieto $^{5}$, K. Nilsson ${ }^{10,33}$, R. Orito ${ }^{13}$, I. Oya ${ }^{5}$, R. Paoletti ${ }^{3}$, J. M. Paredes ${ }^{14}$,

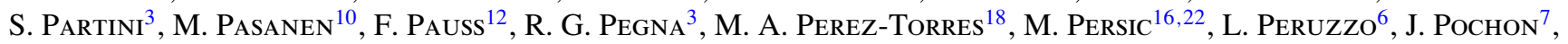

F. Prada ${ }^{18}$, P. G. Prada Moroni ${ }^{3}$, E. Prandini ${ }^{6}$, N. Puchades ${ }^{1}$, I. Puljak ${ }^{19}$, I. Reichardt ${ }^{1}$, R. Reinthal ${ }^{10}$, W. Rhode ${ }^{4}$, M. Ribó ${ }^{14}$, J. Rico ${ }^{1,23}$, S. RÜGamer ${ }^{15}$, A. SAGgion ${ }^{6}$, K. SAIto $^{13}$, T. Y. SaIto ${ }^{13}$, M. Salvati ${ }^{2}$, M. SÁnchez-Conde ${ }^{7,8}$,

K. Satalecka ${ }^{11}$, V. Scalzotto ${ }^{6}$, V. Scapin ${ }^{16}$, C. Schultz ${ }^{6}$, T. Schweizer ${ }^{13}$, M. ShaYduk ${ }^{13}$, S. N. Shore ${ }^{24}$, A. Sierpowska-BartosiK ${ }^{9}$, A. Sillanpä̈̈ ${ }^{10}$, J. Sitarek ${ }^{9,13,32}$, D. Sobczynska ${ }^{9}$, F. Spanier ${ }^{15}$, S. Spiro ${ }^{2}$, A. Stamerra ${ }^{3}$, B. Steinke ${ }^{13}$, J. Storz ${ }^{15}$, N. Strah ${ }^{4}$, J. C. Struebig ${ }^{15}$, T. Suric ${ }^{19}$, L. TAKalo ${ }^{10}$, F. TaveCchio ${ }^{2}$, P. Temnikov ${ }^{21}$, T. Terzić ${ }^{19}$, D. Tescaro ${ }^{1}$, M. Teshima ${ }^{13}$, D. F. Torres ${ }^{17}, 23$, H. Vankov ${ }^{21}$, R. M. Wagner ${ }^{13}$, Q. Weitzel ${ }^{12}$, V. Zabalza ${ }^{14}$, F. ZANDANEL ${ }^{18,32}$, AND R. ZANIN ${ }^{1}$

(The MAGIC COLLABORATION)

AND

A. Neronov ${ }^{25}$, C. Pfrommer ${ }^{26,27}$, A. PinzKe ${ }^{28}$, and D. V. Semikoz ${ }^{29}$

${ }^{1}$ IFAE, Edifici Cn., Campus UAB, E-08193 Bellaterra, Spain

${ }^{2}$ INAF National Institute for Astrophysics, I-00136 Rome, Italy

${ }^{3}$ Università di Siena, and INFN Pisa, I-53100 Siena, Italy

${ }^{4}$ Technische Universität Dortmund, D-44221 Dortmund, Germany

${ }^{5}$ Universidad Complutense, E-28040 Madrid, Spain

${ }^{6}$ Università di Padova and INFN, I-35131 Padova, Italy

${ }^{7}$ Inst. de Astrofísica de Canarias, E-38200 La Laguna, Tenerife, Spain

${ }^{8}$ Depto. de Astrofísica, Universidad de La Laguna, E-38206 La Laguna, Spain

${ }^{9}$ University of Łódź, PL-90236 Lodz, Poland

${ }^{10}$ Tuorla Observatory, University of Turku, FI-21500 Piikkiö, Finland

${ }^{11}$ Deutsches Elektronen-Synchrotron (DESY), D-15738 Zeuthen, Germany ${ }^{12}$ ETH Zurich, CH-8093, Switzerland

${ }^{13}$ Max-Planck-Institut für Physik, D-80805 München, Germany

${ }^{14}$ Universitat de Barcelona (ICC/IEEC), E-08028 Barcelona, Spain

${ }^{15}$ Universität Würzburg, D-97074 Würzburg, Germany

${ }^{16}$ Università di Udine, and INFN Trieste, I-33100 Udine, Italy

${ }^{17}$ Institut de Ciències de l'Espai (IEEC-CSIC), E-08193 Bellaterra, Spain

${ }^{18}$ Inst. de Astrofísica de Andalucía (CSIC), E-18080 Granada, Spain

${ }^{19}$ Croatian MAGIC Consortium, Institute R. Boskovic, University of Rijeka and University of Split, HR-10000 Zagreb, Croatia

${ }^{20}$ Universitat Autònoma de Barcelona, E-08193 Bellaterra, Spain

${ }^{21}$ Inst. for Nucl. Research and Nucl. Energy, BG-1784 Sofia, Bulgaria

${ }^{22}$ INAF/Osservatorio Astronomico and INFN, I-34143 Trieste, Italy

${ }^{23}$ ICREA, E-08010 Barcelona, Spain

${ }^{24}$ Università di Pisa, and INFN Pisa, I-56126 Pisa, Italy

${ }^{25}$ SDC Data Center for Astrophysics, Geneva Observatory, Chemin d'Ecogia 16, 1290 Versoix, Switzerland

${ }^{26}$ HITS, Schloss-Wolfsbrunnenweg 33, 69118 Heidelberg, Germany

${ }^{27}$ CITA, University of Toronto, M5S 3H8 Toronto, ON, Canada

${ }^{28}$ Stockholm University, SE - 10691 Stockholm, Sweden

${ }^{29}$ APC, 10 rue Alice Domon et Leonie Duquet, F-75205 Paris Cedex 13, France

Received 2010 September 11; accepted 2010 September 30; published 2010 October 18

\section{ABSTRACT}

We report on the detection with the MAGIC telescopes of very high energy (VHE) $\gamma$-rays from IC 310, a head-tail radio galaxy in the Perseus galaxy cluster, observed during the interval 2008 November to 2010 February. The Fermi satellite has also detected this galaxy. The source is detected by MAGIC at a high statistical significance of $7.6 \sigma$ in $20.6 \mathrm{hr}$ of stereo data. The observed spectral energy distribution is flat with a differential spectral index of $-2.00 \pm 0.14$. The mean flux above $300 \mathrm{GeV}$, between 2009 October and 2010 February, $(3.1 \pm 0.5) \times 10^{-12} \mathrm{~cm}^{-2} \mathrm{~s}^{-1}$, corresponds to $(2.5 \pm 0.4) \%$ of Crab Nebula units. Only an upper limit, of $1.9 \%$ of 
Crab Nebula units above $300 \mathrm{GeV}$, was obtained with the 2008 data. This, together with strong hints ( $>3 \sigma)$ of flares in the middle of 2009 October and November, implies that the emission is variable. The MAGIC results favor a scenario with the VHE emission originating from the inner jet close to the central engine. More complicated models than a simple one-zone synchrotron self-Compton (SSC) scenario, e.g., multi-zone SSC, external Compton, or hadronic, may be required to explain the very flat spectrum and its extension over more than three orders of magnitude in energy.

Key words: galaxies: active - galaxies: individual (IC 310) - gamma rays: galaxies

\section{INTRODUCTION}

Most of the presently known extragalactic very high energy (VHE, above $300 \mathrm{GeV}) \gamma$-ray emitters $(\sim 40)$ are blazars. So far only two radio galaxies, M 87 (Aharonian et al. 2003) and Cen A (Aharonian et al. 2009), and two starburst galaxies, NGC 253 (Acero et al. 2009) and M82 (Karlsson et al. 2009), have been clearly identified in this energy range.

IC 310 (redshift $z=0.019$ ) is a head-tail radio galaxy located in the Perseus cluster at 0.6 (corresponding to $\sim 1 \mathrm{Mpc}$ ) from the cluster's central galaxy, NGC 1275. Head-tail radio galaxies display a radio morphology consisting of a bright head, close to the optical galaxy and a fainter, elongated tail. In the standard explanation, the jets are bent toward one direction creating the "head" structure. At larger distances they fan out in a characteristic tail that extends over many tens to hundreds of kpc. When the flow of intracluster medium (ICM) impacting these galaxies (in their rest frame) is supersonic (Mach number larger than 1), the ram pressure of the ICM causes the jets to bend (Begelman et al. 1979). If the flow is transsonic (Mach number $\sim 1$ ), the thermal pressure gradient of the interstellar medium of these galaxies, due to their motion through the ICM, determines the bending (Jones \& Owen 1979). In this last model, the inflow is decelerated and heated by a bow shock in front of the galaxy, which also generates a turbulent wake that re-accelerates the relativistic particle population in the tail and illuminates the tail.

The radio contours of IC 310 show an extended emission, pointing away from the direction of NGC 1275. The length of this tail measured in radio varies between 0.12 and 0.27 (Sijbring \& de Bruyn 1998; Lal \& Rao 2005). The X-ray image of IC 310 observed by XMM-Newton is compatible with a pointlike emission from the core and with no X-ray emission from its extended radio structure (Sato et al. 2005). Interestingly, Sato et al. (2005) also showed that the X-ray emission may originate from the central active galactic nucleus (AGN) of the BL Lac-type object. Other observed characteristics of IC 310 (e.g., no strong emission lines, spectral indexes in radio and $\mathrm{X}$-ray) suggest that it may also be a dim (weakly beamed) blazar (Rector et al. 1999).

The LAT instrument on board the Fermi satellite (Atwood et al. 2009) has recently detected IC 310 (Neronov et al. 2010) with five (three) photons above $30 \mathrm{GeV}$ (above $100 \mathrm{GeV}$ ). At lower energy (i.e., from $100 \mathrm{MeV}$ to $1 \mathrm{GeV}$ ), only the cluster's central galaxy, NGC 1275, is visible (Abdo et al. 2009; Neronov et al. 2010).

In this Letter, we present the results of recent observations of the Perseus cluster performed with the Major Atmospheric Gamma Imaging Cherenkov (MAGIC) telescopes and the detection of IC 310 (ATel 2510).

\footnotetext{
30 Supported by INFN Padova.

${ }^{31}$ Now at Centro de Investigaciones Energéticas, Medioambientales y Tecnológicas.

32 Send offprint requests to J. Sitarek (jsitarek@mppmu.mpg.de), F. Zandanel (fabio@iaa.es), and S. Lombardi (slombard@pd.infn.it).

${ }^{33}$ Now at Finnish Centre for Astronomy with ESO (FINCA), Turku, Finland.
}

\section{OBSERVATION AND ANALYSIS}

MAGIC consists of two $17 \mathrm{~m}$ Imaging Atmospheric Cherenkov Telescopes (IACTs) located at the Roque de los Muchachos, Canary Island of La Palma $\left(28^{\circ} \mathrm{N}, 18^{\circ} \mathrm{W}\right)$, at the height of $2200 \mathrm{~m}$ a.s.l. The commissioning of the second MAGIC telescope was finished in 2009, and since the end of 2009 both telescopes work together in stereo mode (Cortina et al. 2009). The stereo observations provide an excellent sensitivity of $<1 \%$ of the Crab flux (C.U.) ${ }^{34}$ in the medium energy range in $50 \mathrm{hr}$ of observations (Colin et al. 2009).

The MAGIC I telescope was used to observe the Perseus cluster for a total of $94 \mathrm{hr}$ between 2008 November and 2010 February. The analysis of $33.4 \mathrm{hr}$ of data taken in 2008 was presented in Aleksić et al. (2010a); it focused on the physics of the Perseus cluster and NGC 1275. The skymap presented in Aleksić et al. (2010a) does not show significant excesses in the IC 310 position. ${ }^{35}$ Since the end of 2009 October the second MAGIC telescope was also taking data, allowing the stereoscopic analysis.

Observations were performed in the so-called wobble mode, i.e., with data equally split into two pointing positions offset by 0.4 from the direction of NGC 1275 . IC 310 was in the field of view at the angular distance of 0.25 and $1^{\circ}$ for individual wobble positions. Since the $\gamma$-ray collection area in the latter case is significantly lower (by a factor of $\sim 3$ ), only data with 0.25 offset were used for the signal search and for obtaining the spectrum and the light curve.

We separately analyzed the MAGIC I single telescope (hereafter mono) and stereo data. The mono and stereo are only partially independent systems, differing in the analysis method, thus there can be some residual systematic error between them. For single telescope observations, the systematic error in the determination of the flux is $\sim 30 \%$ (Albert et al. 2008a). Additionally, IC 310 was not observed in the standard wobble observation mode; this increases the systematic error from the background estimate. The mono data are treated with the standard MAGIC I analysis chain (Albert et al. 2008a; Aliu et al. 2009). In the analysis of the stereo data we took advantage of the impact parameters with respect to each telescope and the height of the shower maximum. Those stereo parameters improve the gamma/hadron separation and the energy reconstruction (Aharonian et al. 1997; MAGIC stereo performance paper 2010, in preparation). We used a new method to reconstruct the arrival directions that improves the angular resolution and the sensitivity of the MAGIC telescopes. The method is based on the "DISP RF" technique (see Aleksić et al. 2010b for details), adapted to the stereo observations. The estimate of an arrival direction is performed independently for each telescope using the shape and

\footnotetext{
34 Hereafter C.U. stands for Crab Units, defined as the fraction of the Crab Nebula flux defined by Equation (2) of Albert et al. (2008a), that corresponds, e.g., for energies above $300 \mathrm{GeV}$, to $12.4 \times 10^{-11} \mathrm{~cm}^{-2} \mathrm{~s}^{-1}$.

35 Throughout this Letter, we refer to the IC 310 position as the position reported by the NASA Extragalactic Database (NED) from 2MASS (2003) catalog.
} 


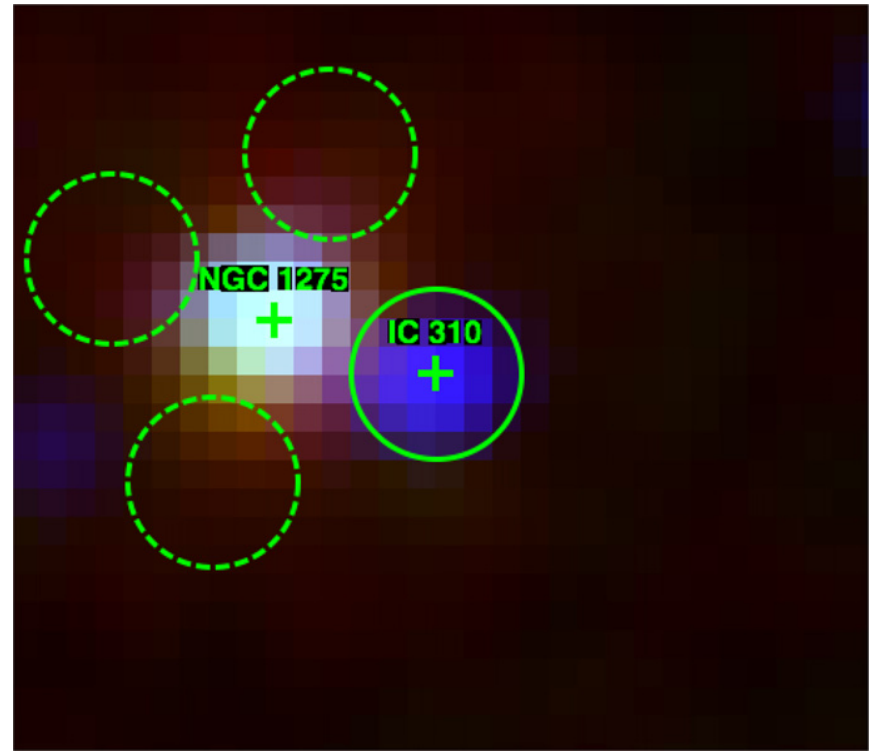

Figure 1. Fermi-LAT count rate image of the IC 310 region in three energy bands: $0.3-3 \mathrm{GeV}$ (red), 3-30 GeV (green), and 30-300 GeV (blue). The position of IC 310 and NGC 1275 is shown with green crosses. Solid (dashed) circles show the signal (background) integration regions used for obtaining the Fermi-LAT spectrum.

time information of a corresponding image. In order to obtain the best performance, those are combined with the crossing point of the main axes of the images from both telescopes.

We also analyzed Fermi data taken during the period between 2008 August 4 and 2010 July 15 following the approach of Neronov et al. (2010). The data were filtered with gtselect tool, and the Fermi exposure at the source position was calculated using gtexposure tool. ${ }^{36}$ Only events of "diffuse" class were retained in the analysis. We obtained the spectrum of IC 310 in the 2-200 GeV energy band in two different ways. First, we performed a spectral fit using the standard Fermi unbinned likelihood analysis, taking into account all sources from the first year Fermi catalog (Abdo et al. 2010) within $10^{\circ}$ of IC 310. We then obtain spectral points by extracting photon counts from the circle of radius 0.3 centered on the source. Taking into account the proximity to a bright, nearby source (radio galaxy NGC 1275), we estimated the background by taking three apertures at 0.6 away from NGC 1275 (see Figure 1).

36 See http://fermi.gsfc.nasa.gov/ssc/data/analysis/scitools/ for details on the Fermi analysis, we used the software version v9r15p2.

\section{RESULTS}

After the data quality check, we obtained a sample for the period from 2009 October to 2010 February, of $20.6 \mathrm{hr}$ of MAGIC stereo data. The theta ${ }^{2}$ (squared distance between true and reconstructed source position; see, e.g., Daum et al. 1997) distribution of the signal coming from IC 310 and the background estimation are shown in Figure 2 (left panel). We found an excess of 106 events, corresponding to a $7.6 \sigma$ significance (calculated according to the prescription by $\mathrm{Li} \&$ Ma 1983, Equation (17)).

The source was also detected in the $27.5 \mathrm{hr}$ of mono data (2009 September to 2010 February) with a significance of $8.6 \sigma$. Note that since part of the MAGIC I data set is also used in the stereo analysis, the two significances are not completely independent. The corresponding alpha (the angle between the main axis of the $\gamma$-ray-induced shower image and the direction to the true source position; see, e.g., Albert et al. 2008a) distribution is also shown in Figure 2 (middle panel). The different signal significance obtained in stereo $(7.6 \sigma)$ is similar to the one of mono scaled to the same observation time $(8.6 \sigma \times \sqrt{20.6 \mathrm{hr} / 27.5 \mathrm{hr}}=7.5 \sigma)$. This is because the mono data have been taken over a longer time period, including a higher emission state in 2009 October (see below). Moreover, in significance calculated according to $\mathrm{Li} \& \mathrm{Ma}$ (1983) the background is estimated using both $\mathrm{ON}$ and OFF measurements. Thus for a high signal/background ratio (as in the case of excellent gamma/hadron separation obtained in stereo observations), the background is overestimated and this lowers the significance. A perfectly known background of $N_{\mathrm{gbg}}$ events will fluctuate with a standard deviation of $\sqrt{N_{\mathrm{bg}}}$, thus a significance of a weak signal of $N_{\mathrm{ex}}$ events can be approximated by $N_{\text {ex }} / \sqrt{N_{\mathrm{bg}}}$. This formula scaled to the same observation time gives a higher value for stereo, 10.2, than for mono, 9.2, observations.

It is interesting to note that the $11.2 \mathrm{hr}$ of good quality, mono data taken at the end of 2008 do not show any significant excess at the position of IC 310 (see Figure 2, right panel). These data yield an upper limit for the flux $F(>300 \mathrm{GeV})<1.9 \%$ C.U. (calculated using the Rolke et al. 2005 method with $95 \%$ of confidence level and assuming $30 \%$ systematic error in absolute flux level).

At energies above $400 \mathrm{GeV}$ the MAGIC telescopes working together in stereo have a point-spread function of $\sim 0.06$, defined as a $40 \%$ containment radius, corresponding to a $\sigma$ of a two-dimensional Gaussian. In Figure 3, we show the significance map of the Perseus cluster region above $400 \mathrm{GeV}$. The bright spot is consistent with the position of the IC 310 .
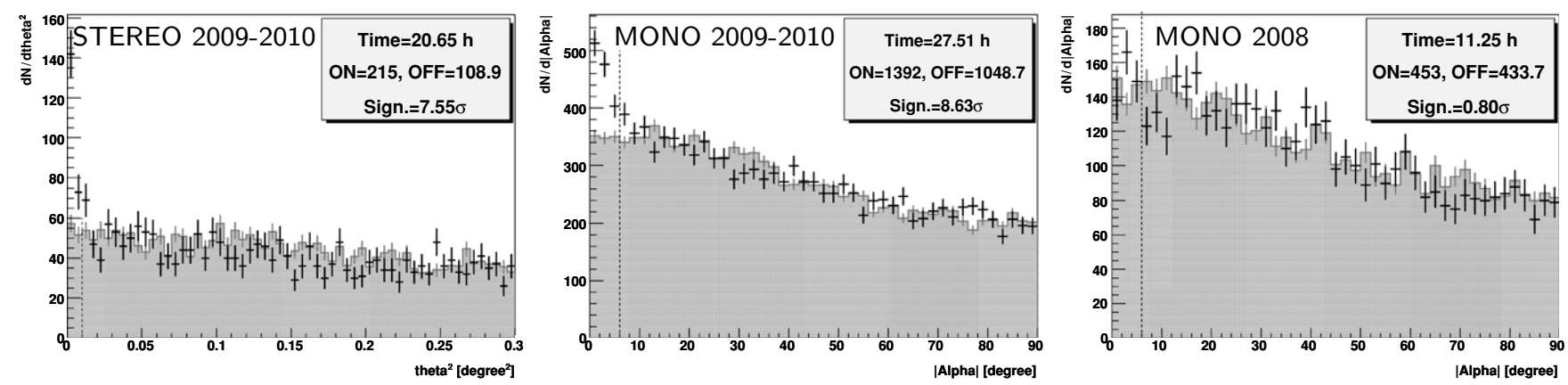

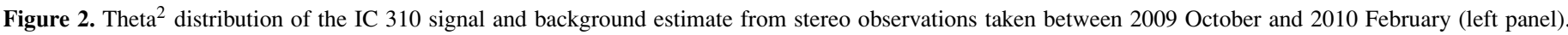

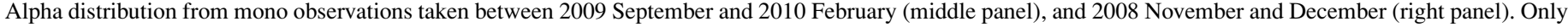

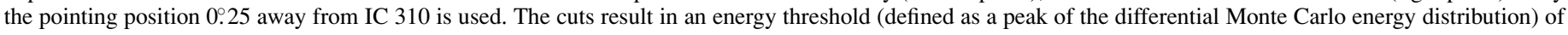
$\sim 260 \mathrm{GeV}$ for both mono and stereo data (see the text for details). 


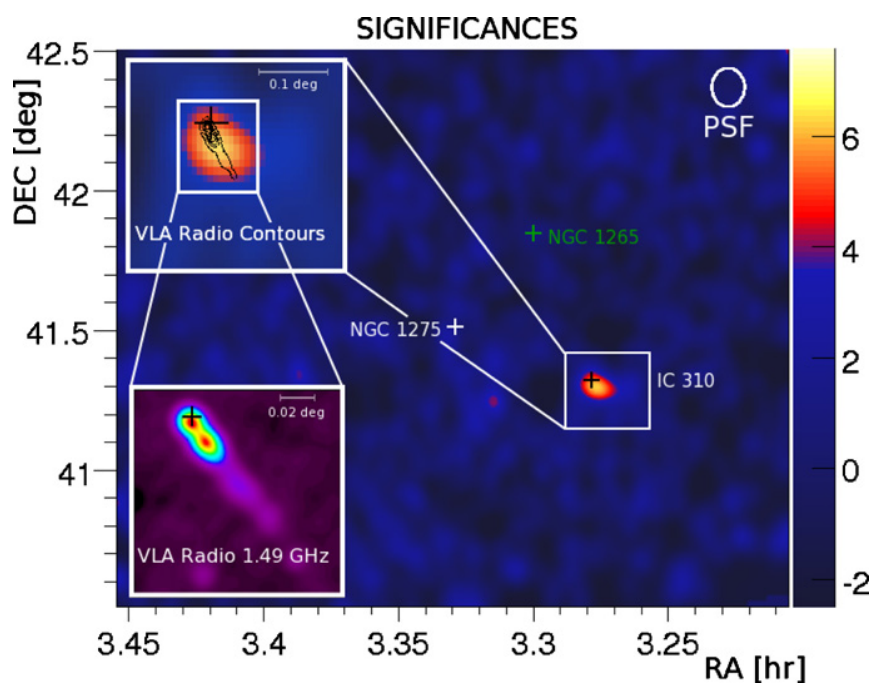

Figure 3. Significance skymap from the MAGIC stereo observation ( $42 \mathrm{hr}$; both pointing positions) for energies above $400 \mathrm{GeV}$. We also show an enlargement of the IC 310 region overlaid with the NVSS (NRAO VLA Sky Survey at $1.49 \mathrm{GHz}$; Condon \& Broderick 1988) contours (top left inserted panel) and the corresponding NVSS image (bottom left inserted panel). The NVSS data were obtained with Aladin (Bonnarel et al. 2000). Positions of IC 310, NGC 1275, and NGC 1265 are marked with black, white, and green crosses, respectively.

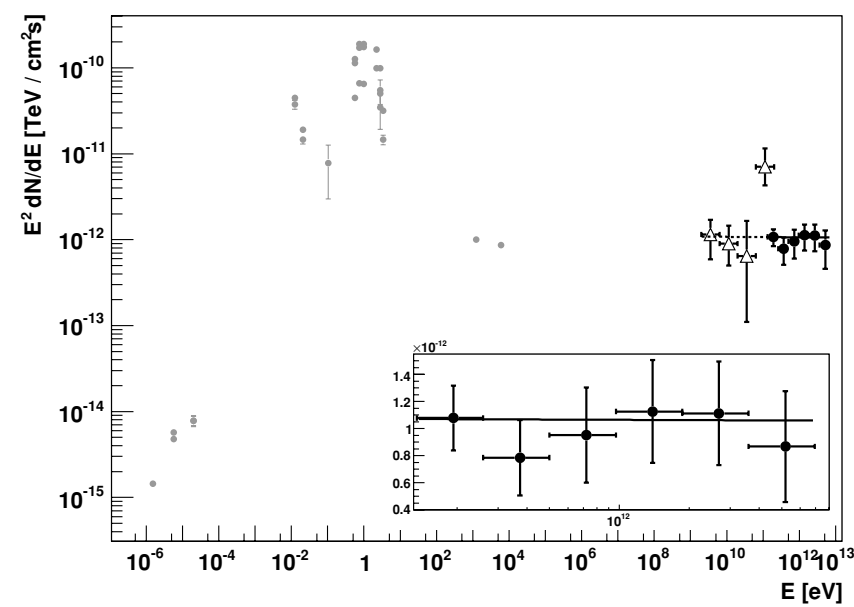

Figure 4. SED of IC 310 obtained with $20.6 \mathrm{hr}$ of the MAGIC stereo data (full circles). Open triangles show the flux measurements from the Fermi-LAT from its first two years of operation. Archival X-ray (Sato et al. 2005), optical (Zwicky \& Kowal 1968; de Vaucouleurs et al. 1991), IR (2MASS 2003; Knapp et al. 1989; IRAS 1988), and radio (Gregory \& Condon 1991; Becker et al. 1991; Condon et al. 2002; White \& Becker 1992; Douglas et al. 1996) data obtained from the NED database are shown with gray dots. The solid line shows a power-law fit to the MAGIC data, and the dotted line is its extrapolation to $\mathrm{GeV}$ energies. We also show a zoom-in of the MAGIC points.

In the panels inserted in Figure 3, we also show archival (nonsimultaneous) IC 310 Very Large Array (VLA) radio data (Condon \& Broderick 1988).

The MAGIC stereo observations reveal a flat spectral energy distribution (SED) between $150 \mathrm{GeV}$ and $7 \mathrm{TeV}$ without any visible curvature or cutoff (see Figure 4 ). The differential flux in units of $\mathrm{cm}^{-2} \mathrm{~s}^{-1} \mathrm{TeV}^{-1}$ is well described $\left(\chi^{2} / n_{\mathrm{dof}}=2.3 / 4\right)$ by a pure power law:

$$
d N / d E=(1.1 \pm 0.2) \times 10^{-12}(E / \mathrm{TeV})^{-2.00 \pm 0.14} .
$$

The mean $\gamma$-ray flux above $300 \mathrm{GeV}$ obtained from the stereo observations between 2009 October and 2010 February is $(3.1 \pm 0.5) \times 10^{-12} \mathrm{~cm}^{-2} \mathrm{~s}^{-1}$, corresponding to $(2.5 \pm 0.4) \%$

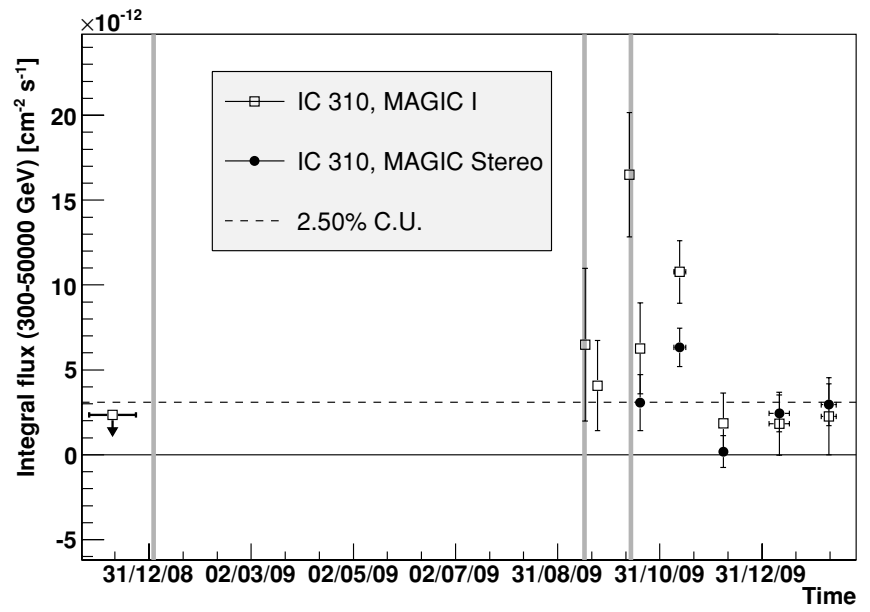

Figure 5. Light curve (in 10 day bins) of the $\gamma$-ray emission above $300 \mathrm{GeV}$ obtained with the mono (open squares) and the stereo (full circles) MAGIC data. The open square with an arrow is the upper limit on the emission in 2008 November and December. Vertical gray lines show the arrival times of $>100 \mathrm{GeV}$ photons from the Fermi-LAT instrument. The horizontal dashed line is a flux level of $2.5 \%$ C.U.

C.U. Comparing this with the upper limit from the 2008 data suggests variability of IC 310 on a one-year timescale.

The light curves of IC $310 \gamma$-ray emission above $300 \mathrm{GeV}$ obtained both with the mono and stereo data are presented in Figure 5. Hints of variability can be seen in the data. Fitting the individual light curves assuming constant flux yields $\chi^{2} / n_{\mathrm{dof}}=$ 27.6/7 (for mono, corresponding to $3.5 \sigma$ ) and 17.5/4 (for stereo, corresponding to $3.0 \sigma$ ). The largest deviations from the mean value are for the intervals 2009 October 13 and $14(3.1 \sigma$

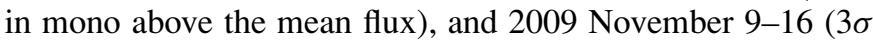
in mono, $3.2 \sigma$ in stereo above the mean flux).

Until 2010 February, the Fermi-LAT instrument observed only three photons with energies above $100 \mathrm{GeV}$ from the direction of IC 310 (Neronov et al. 2010). It is interesting to note that one of those $\gamma$-rays was observed on 15 th of October, nearly coincident with the higher flux seen in mono.

The standard Fermi likelihood analysis gave a "Test Statistics" value of 79 from IC 310 above $1 \mathrm{GeV}$ (corresponding to a $\sim 9 \sigma$ detection). ${ }^{37}$ Assuming a simple power law for the spectrum, we obtain the differential flux $d N / d E=$ $(9.5 \pm 2.9) \times 10^{-9}(E / 10 \mathrm{GeV})^{-1.58 \pm 0.25} \mathrm{~cm}^{-2} \mathrm{~s}^{-1} \mathrm{TeV}^{-1}$. The Fermi spectral index is very hard, mostly due to the last point.

\section{CONCLUSIONS AND DISCUSSION}

The MAGIC telescopes have detected VHE $\gamma$-ray emission with high statistical significance from the direction of IC 310 with a mean $\gamma$-ray flux above $300 \mathrm{GeV}$, between 2009 October and 2010 February, of $(2.5 \pm 0.4) \%$ C.U. The source seems to be variable on the timescales from weeks to about one year. No significant excesses were observed in the 2008 mono data, and flares are possibly present at the $\gtrsim 3 \sigma$ level in 2009 October and November. The stereo observations yield a spectrum that is well fitted by a simple power law with a spectral index of $-2.00 \pm 0.14$.

The MAGIC angular resolution is not sufficient to determine the location of the VHE emission region within the radio galaxy.

\footnotetext{
37 Note that this significance is not calculated according to the $\mathrm{Li} \& \mathrm{Ma}$
} (1983) method, see Fermi analysis description (footnote 35). 
Therefore, it is not clear whether the observed $\gamma$-ray emission is connected with the tail of the source or if it is produced at the base of the jet, close to the central engine of the source (as in blazars). The strong indications of variability disfavor the $\gamma$-ray production at the bow shock, discussed by Neronov et al. (2010), because in this case the emission should be steady on timescales of thousands of years. Variability with a timescale of a year (a week) constrains the size of emission region to be $\lesssim 10^{18} \mathrm{~cm}$ $\left(\lesssim 2 \times 10^{16} \mathrm{~cm}\right)$ across, assuming no Doppler boosting of the flux, which is much smaller than the total size of the tail $\left(\sim 10^{24} \mathrm{~cm}\right)$. Additionally, one can estimate the mass of the central black hole, $M_{\mathrm{BH}}$, of an active galaxy using the correlation between black hole mass and the central velocity dispersion of the host galaxy (Tremaine et al. 2002). The measured velocity dispersion in IC $310\left(230 \mathrm{~km} \mathrm{~s}^{-1}\right.$; McElroy 1995) yields $M_{\mathrm{BH}}=2.4 \times 10^{8} M_{\odot}$, corresponding to a Schwarzschild radius of $R_{\mathrm{SH}}=7 \times 10^{13} \mathrm{~cm}$. This indicates that the most probable location of the $\gamma$-ray emission region is in the innermost part of the jet (as, e.g., for M 87; see Acciari et al. 2009a).

The extrapolation of the IC 310 spectrum obtained with the MAGIC telescopes is in good agreement with the Fermi spectrum below $60 \mathrm{GeV}$. On the other hand, there is a large deviation in the highest energy bin measured by Fermi (see Figure 4). The $\gamma$-ray flux from our observations in this energy bin predicts 0.6 photons, while 4 photons were observed by Fermi-LAT. Assuming a Poisson distribution, the probability of obtaining $\geqslant 4$ photons is $3.4 \times 10^{-3}$ (corresponding to 2.7 standard deviations) so the discrepancy may be a statistical fluctuation. However, the Fermi-LAT, having an energy resolution of $\sim 10 \%$, observed three of the four photons with nearly the same energies $(98.5,105$, and $111 \mathrm{GeV})$. If confirmed by future observations, these events may indicate the presence of a peculiar peak or bump in the IC 310 spectrum given that the remaining FermiLAT data agree with the MAGIC measurement. The detection of such a relatively narrow feature in the spectrum of a radio galaxy may be a clue regarding the particle acceleration mechanism at the base of AGN jets (e.g., "direct" gamma-ray emission during acceleration of particles; Neronov \& Aharonian 2007). This remains a suggestion since the source seems to be variable, and the MAGIC and Fermi data used here were not taken simultaneously.

The combined MAGIC and Fermi spectrum (besides the above mentioned bump) is consistent with a flat $E^{-2}$ spectrum stretching without a break over more than three orders of magnitude in energy $(2 \mathrm{GeV}-7 \mathrm{TeV})$. This is similar to the flat VHE spectra of M 87, another radio galaxy detected at $\mathrm{TeV}$ energies (Aharonian et al. 2006; Albert et al. 2008b; Acciari et al. 2008). Such an extended, flat $E^{-2}$ spectrum is hard to obtain in a simple one-zone synchrotron self-Compton (SSC) model (e.g., Maraschi et al. 1992). Instead, a viable model of emission might be inverse Compton scattering of external IR photon background photons from accretion flow or from the inner jet (see, e.g., Neronov \& Aharonian 2007). Alternatively, a flat spectrum can be produced in the hadronic models (e.g., Mannheim 1993; Mücke et al. 2003). In more complicated, multi-zone leptonic models, the $\mathrm{GeV}-\mathrm{TeV}$ emission of a few slightly shifted inverse Compton peaks can also emulate a flat spectrum (e.g., spine-sheath layer model, Tavecchio \& Ghisellini 2008, or SSC+EC model used for explaining SED of an IBL object W Com, Acciari et al. 2009b). Finally, using the model by Dominguez et al. (2010), we find that the change in the spectrum due to the absorption in the extragalactic background light radiation field is within the error of the spectral slope.
IC 310 and NGC 1265 were the first two head-tail galaxy discovered by astronomers (Ryle \& Windram 1968). Now, IC 310 is the first head-tail radio galaxy detected in the VHE $\gamma$-rays by ground-based telescopes as well as by the Fermi satellite. Additionally, it is also the first source discovered above $300 \mathrm{GeV}$ by the MAGIC telescopes working together in stereo mode. This detection is also important for the VHE study of the Perseus cluster of galaxies. It may imply a substantial injection of high energy particles in the ICM by a non-central AGN. Those particles might have important consequences on the possible cluster $\gamma$-ray emission due to cosmic-ray acceleration (Aleksić et al. 2010a). The VHE IC 310 detection significantly enriches our knowledge of the $\gamma$-ray universe.

We thank the Instituto de Astrofísica de Canarias for the excellent working conditions at the Observatorio del Roque de los Muchachos in La Palma. The support of the German BMBF and MPG, the Italian INFN, the Swiss National Fund SNF, and the Spanish MICINN is gratefully acknowledged. This work was also supported by the Marie Curie program, by the CPAN CSD2007-00042 and MultiDark CSD2009-00064 projects of the Spanish Consolider-Ingenio 2010 programme, by grant DO02-353 of the Bulgarian NSF, by grant 127740 of the Academy of Finland, by the YIP of the Helmholtz Gemeinschaft, by the DFG Cluster of Excellence "Origin and Structure of the Universe," and by the Polish MNiSzW Grant N N203 390834. This research has made use of the NASA/IPAC Extragalactic Database (NED) which is operated by the Jet Propulsion Laboratory, California Institute of Technology, under contract with the National Aeronautics and Space Administration.

\section{REFERENCES}

Abdo, A. A., et al. 2009, ApJ, 699, 31

Abdo, A. A., et al. 2010, ApJS, 188, 405

Acciari, V. A., et al. 2008, ApJ, 679, 397

Acciari, V. A., et al. 2009a, Science, 325, 444

Acciari, V. A., et al. 2009b, ApJ, 707, 612

Acero, F., et al. 2009, Science, 326, 1080

Aharonian, F. A., Hofmann, W., Konopelko, A. K., \& Völk, H. J. 1997, Astropart. Phys., 6, 343

Aharonian, F., et al. 2003, A\&A, 403, L1

Aharonian, F. A., et al. 2006, Science, 314, 1424

Aharonian, F., et al. 2009, ApJ, 695, L40

Albert, J., et al. 2008a, ApJ, 674, 1037

Albert, J., et al. 2008b, ApJ, 685, L23

Aleksić, J., et al. 2010a, ApJ, 710, 634

Aleksić, J., et al. 2010b, arXiv:1004.1093

Aliu, E., et al. 2009, Astropart. Phys., 30, 293

Atwood, W. B., et al. 2009, ApJ, 697, 1071

Becker, R. H., White, R. L., \& Edwards, A. L. 1991, ApJS, 75, 1

Begelman, M. C., Rees, M. J., \& Blandford, R. D. 1979, Nature, 279, 770

Bonnarel, F., et al. 2000, A\&AS, 143, 33

Colin, P., et al. 2009, Proc. 31st ICRC, arXiv:0907.0960

Condon, J. J., \& Broderick, J. J. 1988, AJ, 96, 30

Condon, J. J., Cotton, W. D., \& Broderick, J. J. 2002, AJ, 124, 675

Cortina, J., Goebel, F., \& Schweizer, T. 2009, Proc. 31st ICRC, arXiv:0907.1211

Daum, A., et al. 1997, Astropart. Phys., 8, 1

de Vaucouleurs, G., de Vaucouleurs, A., Corwin, H. G., Jr., Buta, R. J., Paturel, G., \& Fouque, P. 1991, Third Reference Catalogue of Bright Galaxies (New York: Springer)

Dominguez, A., et al. 2010, arXiv:1007.1459

Douglas, J. N., Bash, F. N., Bozyan, F. A., Torrence, G. W., \& Wolfe, C. 1996, AJ, 111,1945

Gregory, P. C., \& Condon, J. J. 1991, ApJS, 75, 1011

Joint IRAS Science Working Group 1988, Infrared Astronomical Satellite Catalogs, the Point Source Catalog (Washington, DC: NASA)

Jones, T. W., \& Owen, F. N. 1979, ApJ, 234, 818 
Karlsson, N., et al. 2009, Fermi Symp., eConf. Proc. C091122, arXiv:0912.3807

Knapp, G. R., Guhathakurta, P., Kim, D.-W., \& Jura, M. A. 1989, ApJS, 70, 329

Lal, D. V., \& Rao, A. P. 2005, in ASP Conf. Ser. 345, From Clark Lake to the Long Wavelength Array: Bill Erickson's Radio Science, ed. N. Kassim et al (San Francisco, CA: ASP), 294

Li, T.-P., \& Ma, Y.-Q. 1983, ApJ, 272, 317

Mannheim, K. 1993, A\&A, 269, 67

Maraschi, L., Ghisellini, G., \& Celotti, A. 1992, ApJ, 397, L5

McElroy, D. B. 1995, ApJS, 100, 105

Mücke, A., Protheroe, R. J., Engel, R., Rachen, J. P., \& Stanev, T. 2003 Astropart. Phys., 18, 593

Neronov, A., \& Aharonian, F. A. 2007, ApJ, 671, 85

Neronov, A., Semikoz, D. V., \& Vovk, I. 2010, arXiv:1003.4615
Rector, T. A., Stocke, J. T., \& Perlman, E. S. 1999, ApJ, 516, 145

Rolke, W. A., López, A. M., \& Conrad, J. 2005, Nucl. Instrum. Methods Phys. Res. A, 551, 493

Ryle, M., \& Windram, M. D. 1968, MNRAS, 138, 1

Sato, K., et al. 2005, PASJ, 57, 743

Sijbring, D., \& de Bruyn, A. G. 1998, A\&A, 331, 901

Tavecchio, F., \& Ghisellini, G. 2008, MNRAS, 385, L98

Tremaine, S., et al. 2002, ApJ, 574, 740

Two Micron All Sky Survey Team 2003, 2MASS Extended Objects (Pasadena, CA: Caltech/IPAC)

White, R. L., \& Becker, R. H. 1992, ApJS, 79, 331

Zwicky, F., \& Kowal, C. T. 1968, Catalogue of Galaxies and of Clusters of Galaxies, Vol. VI (Pasadena, CA: California Institute of Technology) 